\title{
Application of T-S Fuzzy Neural Network to Pattern Recognition of Corona Discharge
}

\author{
Dianchun Zheng, Renxu Yang, Chuntian Chen, Dawei Zhao* and Jiaxiang Yang \\ Electrical \& Electronic Engineering College, Harbin University of Science and \\ Technology, Harbin 150080 \\ Email:zhengdianchun@126.com
}

\begin{abstract}
Based on the T-S fuzzy neural network, the types of corona discharge are recognized. In this paper, three types of corona discharge test model are designed, then characteristic signals of corona discharge, the maximum, minimum and mean value and including the fractal dimension, are extracted, and then used as the input vectors of neural network, furthermore, accordance with the numbers of input vector and speciality of membership function, the T-S FNN topological structure is investigated and constructed, at the same time, the 4 neurons are selected used as input neuron and the 3 Gaussian functions are choose membership function, then the T-S FNN topological, which is trained and tested for this network, the structure is confirmed as the pattern recognition network in the types of corona discharge, and results show that the network is great effective for pattern recognition of corona discharge.
\end{abstract}

Key words: T-S fuzzy neural network; membership function; corona discharge; pattern recognition

\section{Introduction}

Practical experience shows that there is a relationship between insulation breakdown and partial discharge of high-voltage electrical apparatus in service. Therefore, it is important to detect and identify modes of corona discharges, it is not only offering important data for high-voltage electrical apparatus state monitoring, but also it can forecast prediction for appearing calamity happen in the future. By this time, much effort has been made in applying neural networks to the recognition of corona discharge defect and mode. Many intelligent methods such as fuzzy diagnosis system and neural network diagnosis system as well as expert diagnosis system are applied to monitor electrical insulation systems [1-5]. In reference [1], a time-series approach has been employed to devise neural-network topologies for time-dependent corona discharge pulse pattern recognition applications. Automated recognition of PRPD patterns using a novel complex probabilistic neural network system for the actual classification task [2]. A three-layer artificial neural system with feed forward connections is used for pattern recognition of partial discharge, using BP algorithm as the learning method [4]. The Probabilistic Neural Network (PNN) method has the advantage over multi-layer Neural Network in that it gives rapid training speed and guarantees convergence to a Bayes classifier [5]. In this paper, the fuzzy neural network based on T-S model is presented, and the topology is investigated and designed, then the T-S FNN algorithm is also studied. Original characteristic and fractal characteristic are extracted from the corona discharge signal as the input vector of the network.

\footnotetext{
${ }^{*}$ Corresponding author
} 


\section{Signal Process}

\subsection{Corona Discharge Signal}

In order to simulate corona discharge in the laboratory, 3 types (needle-needle needle-plane, sphere-plane) of model are made in accordance with corona discharge patterns, applying a voltage of $25 \mathrm{KV}$ between the electrodes and sampling corona discharge signals. Figure 1 shows 3 types corona discharge signal.

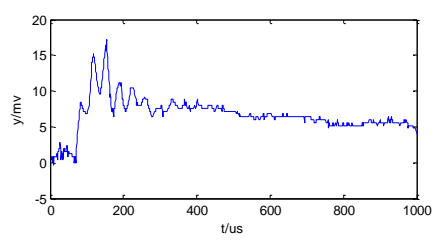

(a) needle-needle

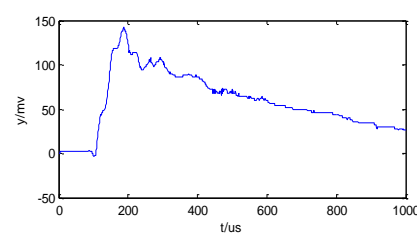

(b) needle-plane

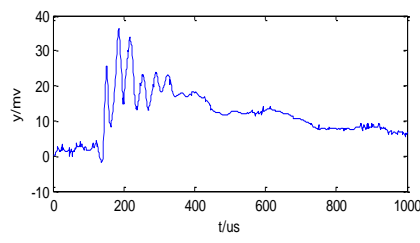

(c) sphere-plane

Figure 1. Corona Discharge Signals

\subsection{Feature Extraction}

Fractal geometry, which is popularized by Mandelbrot [7], recently has gained increasing attention for the segmentation and recognition. In reference [8], fractal dimension $D$ and lacunarity $\Lambda$ have shown to be very successful for partial discharge pattern recognition purposes. Mandelbrot suggests that the number $N$ of boxes of side $r$ necessary to cover a fractal set obeys the power law

$$
N(r)=K r^{-D}
$$

where, $K$ is a constant and $D$ is the fractal dimension of corona discharge signal. Furthermore, the method of estimating $N(r)$ involves the calculation of $f(m, r)$, which is defined as the probability that $m$ points of the set lie in a box of side $r$ centered at any arbitrary point of the fractal set. The dimension $D$ can be estimated from the slope of a straight line, fitting the data $\{\log (r),-\log (N(r))\}$ for several boxes of side $r$.

The original features, which include maximum, minimum and mean, are extracted from the corona discharge signal. According to the waveform of defect model perspective, there is a large difference among different corona discharge signal. Thus original feature can act as the input vector of the network. Original feature and fractal dimension are shown in Table 1.

Table 1. Original and Fractal Features

\begin{tabular}{ccccc}
\hline type & maximum & minimum & mean & fractal \\
\hline needle-plane1 & 132 & -5 & 54.4060 & 1.6330 \\
needle-plane2 & 129 & -6 & 54.8860 & 1.6558 \\
needle-needle1 & 17 & -0.4 & 6.72760 & 1.5299 \\
needle-needle2 & 16 & -1 & 6.55780 & 1.5849 \\
sphere-plane1 & 36 & -2 & 11.4140 & 1.7036 \\
sphere-plane2 & 35 & -2 & 11.1532 & 1.7092 \\
\hline
\end{tabular}




\section{T-S Fuzzy Neural Network}

\subsection{T-S Type Fuzzy Model}

The T-S type fuzzy model, proposed by Takagi and Sugeno, is described by fuzzy IF-THEN rules. The rule of T-S fuzzy neural network is described as follows:

If $x$ is $A_{i}$, and $y$ is $B_{i}$, then $z=p_{i} x+q_{i} y+r_{i}$, where $A_{i}$ and $B_{i}$ are the grade of membership, $p, q, r$ are parameters.

\subsection{T-S FNN Configuration}

The fuzzy neural network based on the T-S model not only can easily express the complex system, but also has logical reasoning ability and the self-study ability, which make the fuzzy neural network have a better performance to recognize defect model. T-S fuzzy neural network configuration is shown in Fig.2. The basic function in each layer is introduced in the following:

$$
\text { Layer 1: } f^{(1)}=x_{i} \quad(i=1,2, \ldots, n)
$$

For every node in this layer is connected directly to the input vectors $X\left(X=\left[x_{1}, x_{2}, \ldots, x_{n}\right]^{T}\right)$, its role is to transmit $X$ to next layer. The neuron number is $n$.

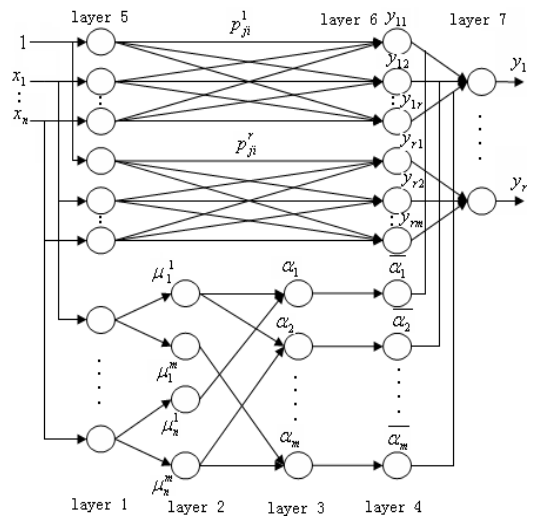

Figure 2. T-S FNN Configuration

Layer2:

$$
\mu_{i}^{s_{i}}=\mu_{A}^{s_{i}}\left(x_{i}\right)\left(i=1,2, \ldots, n ; s_{i}=1,2, \ldots, m_{i}\right)
$$

where, $\mu_{i}^{s_{i}}$ is the membership function, $n$ is the dimension of the input vector, $m_{i}$ is the fuzzy partition of $x_{i}$, the neuron number is $\sum_{i=1}^{n} m_{i}$.

Layer 3:

$$
\alpha_{i}=\min \left(\mu_{1}^{s_{i j}}, \mu_{2}^{s_{2 j}}, \ldots, \mu_{n}^{s_{3 j}}\right)
$$

or

$$
\alpha_{i}=\mu_{1}^{s_{i j}} * \mu_{2}^{s_{2 j}} * \ldots * \mu_{n}^{s_{3 j}}
$$

$$
\begin{aligned}
& s_{1 j} \in\left\{1,2, \ldots, m_{1}\right\}, s_{2 j} \in\left\{1,2, \ldots, m_{2}\right\}, \ldots, \\
& s_{n j} \in\left\{1,2, \ldots, m_{n}\right\}, j=1,2, . ., m, m=\prod_{i=1}^{n} m_{i}
\end{aligned}
$$

where, $\alpha_{i}$ is the grade of membership of every rule, the neuron number is $m$

Layer4: 


$$
\overline{\alpha_{j}}=\alpha_{j} / \sum_{i=1}^{m} \alpha_{i}(j=1,2, \ldots, m)
$$

where $\overline{\alpha_{j}}$ is the normally of the grade of membership for every rule, the neuron number is $m$.

Layer5:

$$
f^{(5)}=x_{i} \quad(i=1,2, \ldots, n) ; x_{0}=1
$$

Layer6:

$$
y_{k j}=p_{j 0}^{k} * x_{0}+p_{j 1}^{k} * x_{1}+\ldots+p_{j n}^{k} * x_{n}=\sum_{l=0}^{n} p_{j l}^{k} * x_{l}
$$

where, $k=1,2, . ., r ; j=1,2, . ., m ; \quad x_{0}=1, \quad p_{j l}$ is the connection weight of the network, $y_{k j}$ is the output value of every rule, the neuron number is $m * r$.

$$
\text { Layer7: } \quad y_{k}=\sum \bar{\alpha}_{j} y_{k j} \quad(k=1,2, . ., r)
$$

where, $y_{k}$ are the state vectors, which represent the types of the defect model.

In order to realize the learning of neural networks, network is converted to ANFIS (Adaptive Network based Fuzzy inference System), used BP learning algorithm and LS learning algorithm to update parameter.

\subsection{Membership Function and Topology}

According to the learning rule, the number of membership function should be at least 2 . However, while the number is more than or equal to 4 , the topology structure of network is quite complex, the nodes suddenly increase, training time is too long. Hence, we only analyze the number of membership function is 2 and 3 in the following.

Step 1 the number of input neuron

Four features (maximum, minimum, mean, fractal dimension) are extracted from corona discharge signals. Hence, the number of input neuron is 4 .

Step 2 membership function.

The number of input membership function is 2 . Figure 4 shows the model structure of ANFIS.

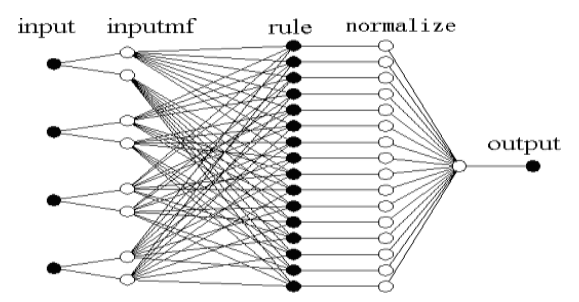

Figure 4. Model Structure of ANFIS

When the membership function is Gaussian function, Figure 5 shows the error change of training. RMS error is $4.0154 \times 10^{-3}$ after training 100 times.

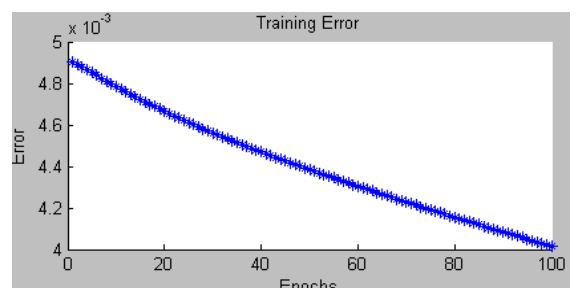

Figure 5. Error Change of Training 
When membership function is bell-type function, Fig.6 shows the error change of training. RMS error is $5.96000 \times 10^{-3}$ after training 100 times.

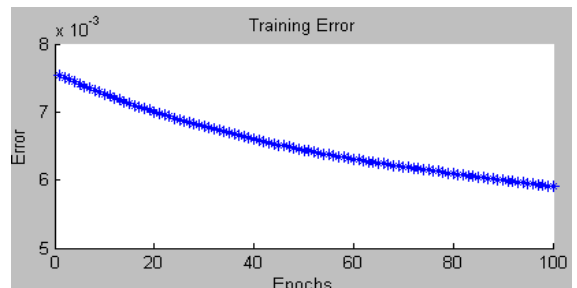

Figure 6. Error Change of Training

1) When the number of input membership function is 3 . Figure 7 shows the model structure of ANFIS.

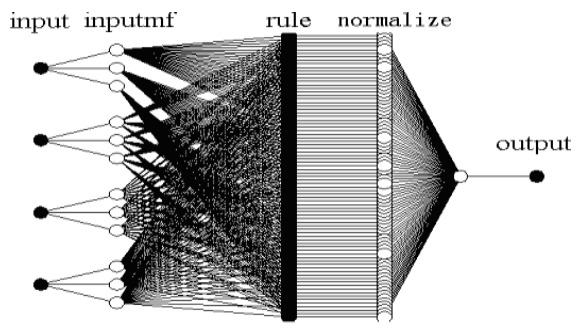

Figure 7. Model Structure of ANFIS

When the membership function is Gaussian function, Figure 8 shows error change of training. RMS error is $3.0231 \times 10^{-4}$ after training 100 times.

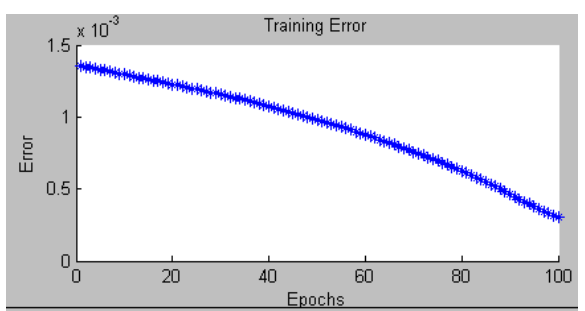

Figure 8. Error Change of Training

When the membership function is bell-type function, Figure 9 shows the error change of training. RMS error is $1.4085 \times 10^{-3}$ after training 100 times.

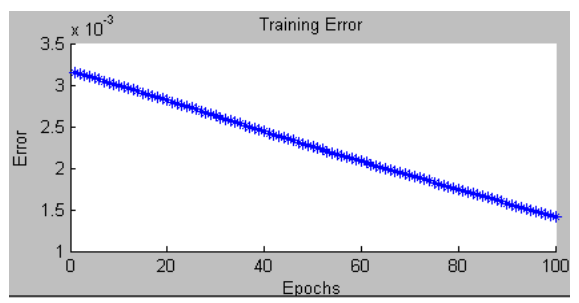

Figure 9. Error Change of Training

According to the above analysis, the ANFIS network, whose membership function is Gaussian function and the number of membership is 3, possesses the performance of fast convergence speed, simple structure and good stability. Hence, this ANFIS network is chosen as the classifier of corona discharge. The topology shows as the Figure 8 . 


\section{Recognition Results}

Training T-S FNN, if training samples come from needle-plane model, then the output equal to 1 ; if training samples come from needle-needle model, then the output equal to 2 ; if training samples come from sphere-plane model, then the output equal to 3. Then, employing the 0.6 norm validates the types of the testing sample [9-10].

Figure 10 and Figure 11 show the error change and output of network after training 100 times. The symbol $\circ$ represents desired output, while $*$ represents the output of network in Figure 11.

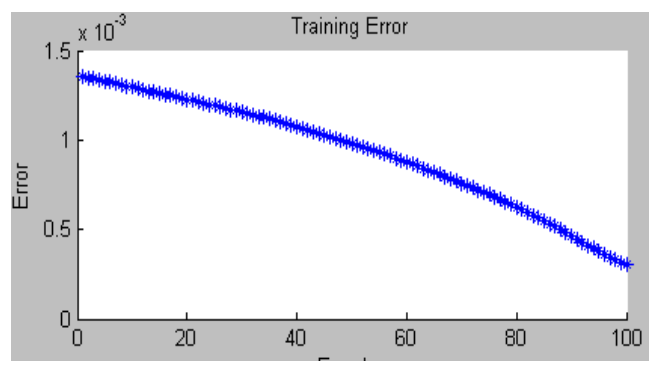

Figure 10. Error Change of Training

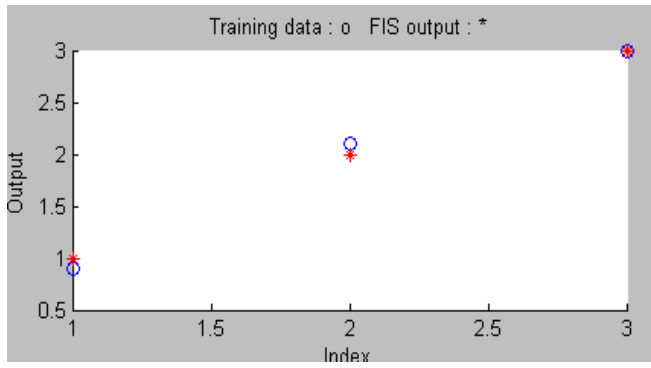

Figure 11. Output of Network

Testing samples are randomly selected, and the classification results are shown in Table 2.

Table 2. Recognition Results of T-S FNN

\begin{tabular}{cccc}
\hline type & needle-needle & needle-plane & sphere-plane \\
\hline $\mathrm{N}$ & 65 & 70 & 65 \\
$\mathrm{~T}$ & 59 & 70 & 61 \\
Rate(\%) & 91 & 100 & 94 \\
\hline
\end{tabular}

It is obvious that in classifying corona discharges T-S FNN shows an excellent performance. It may be due to the training process and available Gaussian function, especially when the recognition rate of needle-plane reaches $100 \%$ performance, while needle-needle and sphere-plane reaches $94 \%$ and $90 \%$, respectively. The reason why the recognition rate of needle-plane reaches $100 \%$ is that the amplitude of the discharge pulse is much larger than others.

\section{Conclusions}

T-S fuzzy neural network is structured and applied for mode classification for corona discharge signals of needle-needle, needle-plane and sphere-plane of experimental model, and the 4 characteristic vectors, which are signal maximum, minimum and mean value and including the fractal dimension, are extracted from corona discharge signals sampled from the experiment models and taken as training and inspection specimen for the T-S fuzzy neural network, meanwhile, the back propagation algorithm and least-square algorithm are also adopted to insure the rapidly convergence and accurately recognition, and the results show that T-S fuzzy neural network has effective performance for recognition for the corona discharge signals come from the experimental model above mentioned, and possesses extensive perspective in the future. However, the proposed T-S FNN is needed to confirm its performance in the case of the on-site application and to improve the recognition rate, then the more effective methods should be think over to obtain the reasonable feature. 


\section{Acknowledgements}

Authors gratefully thank the co-workers for concerning the research project and also acknowledge the Heilongjiang Provincial Education Department's financial support for this research subject (Research Fund of Heilongjiang Provincial Education Department No. 12511z008).

\section{References}

[1] M. M. A. Salama and Bartnikas, "Determination of neural-network topology for partial discharge pulse pattern recognition", IEEE Trans. Neural Networks, vol. 13, no. 2, (2002), pp. 446-456.

[2] B. Karhtikeyan, S. Gopal and M. Vimala, "Conception of complex probabilistic neural network system for classification of partial discharge patterns using multifarious inputs", IEEE Expert Systems with Applications, vol. 29, no. 4, (2005), pp. 953-963.

[3] P.-H. Chen and H.-G Chen, "Application of back-propagation neural network to power transformer insulation diagnosis", Advances in Neural Networks, LNCS, PART 3, vol. 4493, (2007), pp. 26-34.

[4] G. Wu, H. Xie and H. Ma, "Pattern recognition of in large turbine generators with a neural network system", Properties and Applications of Dielectric Materials, vol. 1, (1997), pp. 252-255.

[5] G. Wu, X. Jiang and H. Xie, "Neural network used for PD pattern recognition with genetic algorithm", Properties and Applications of Dielectric Materials, vol. 1, (2000), pp. 451-454.

[6] B. B. Mandelbrot, "The Fractal Geometry of Nature”, New York, (1983).

[7] L. Satish and W. S. Zaengl, "Can fractal feature be used for recognizing 3-d partial discharge patterns", IEEE Trans. on Diel. Elec., vol. 2, no. 3, (1995), pp. 352-359.

[8] W. Zi-jing, Z. Dian-chun and Z. Zheng-han, "The WFCM Recognition Method of Gas Corona Discharge Based Image Gray Features", Harbin, China, (2010).

[9] J. Lei and Z. De-heng, "Ann Based Discharge Pattern Recognition of Insulation Models of Electrical Transformers", Proceedings of the Chinese Society Forelectrical Engineering, vol. 21, (2001), pp. 21-24.

[10] L. Ling, L. Rui-jin and Z. Quan, "PD Pattern Recognition Using Inter-time", High Voltage Engineering, vol. 33, (2007), pp. 35-39. 
International Journal of Signal Processing, Image Processing and Pattern Recognition Vol.7, No.1 (2014) 